\title{
Meeting report: plant genetics and molecular biology
}

\author{
Jung-Kyun Oh $\cdot$ Je-Chang Woo $\cdot$ Nam-Soo Kim
}

Received: 22 January 2014/ Accepted: 25 January 2014/Published online: 28 February 2014

(C) The Genetics Society of Korea 2014

The Genetics Society of Korea (GSK) Mini Symposium III was held October 252013 at the Mokpo National University, Korea. The topic was Plant Genetics and Molecular Biology. There were two sessions, respectively chaired by Professor Je-Chang Woo (Mokpo National University, Korea), the former GSK president, and Professor ChoongMo Park (Seoul National University, Korea), who will be the next GSK president.

In the first session, two speakers presented their findings. The first speaker, professor Sang-Gu Kim (professor emeritus, Seoul National University, Korea), was the founding member and a former president of the GSK and served as the editor-in-chief of the Korean Journal of Genetics (formerly the Genes \& Genomics). In his talk, entitled "Structure-function studies of a plant tyrosyl-DNA phosphodiesterase provide novel insights into DNA repair mechanisms of Arabidopsis thaliana", professor Kim briefly introduced the role plant genetics has played in the development of the GSK, since the founding of the society and then discussed his previous research and findings. Tyrosyl-DNA phosphodiesterase 1 (TDP1) is a member of the phospholipase D superfamily. It catalyzes the hydrolysis of a phosphodiester bond between a tyrosine residue and the 3'-phosphate of DNA. Professor Kim's group isolated TDP1 from Arabidopsis thaliana (AtTDP), which is an ortholog of yeast and human TDP. AtTDP hydrolyzes

J.-K. Oh · J.-C. Woo

Department of Biology, Mokpo National University, Mokpo, Korea

\section{N.-S. Kim $(\bowtie)$}

Department of Molecular Bioscience, Institute of Bioscience and Biotechnology, Kangwon National University, Chuncheon-si 200-701, Korea

e-mail: kimnamsu@kangwon.ac.kr the $3^{\prime}$-phosphotyrosyl DNA substrate, which is related to repairing DNA damage induced by topoisomerase I in vivo. An analysis of the substrate-saturation kinetics of the full-length AtTDP versus those of a truncated AtTDP mutant lacking the N-terminal SMAD/forkhead-associated domain showed that the kinetic parameters of recombinant AtTDP protein and the AtTDP 1-122 (TDP domain) are equivalent to those of the full-length AtTDP. The loss-offunction AtTDP displayed developmental defects and a dwarf phenotype, which was caused by decreased cell numbers rather than changes in cell sizes. The mutant $t d p$ plants exhibited hypersensitivity to camptothecin, a potent topoisomerase I inhibitor, and rigorous cell death in their cotyledons and rosette leaves, suggesting a failure of DNA damage repair. This was the first clear evidence in plants that AtTDP plays a role in DNA repair.

The second speaker in the first session was professor Jeong-Il Kim (Chonnam National University, Korea). The title of professor Kim's talk was “Development of hyperactive phytochromes from the structure-function study in plant light signaling". Phytochromes are photoreceptors that regulate various aspects of plant growth and development in response to red/far-red light signals from the environment. Red-light absorbing (Pr) phytochromes $(\lambda \max =660 \mathrm{~nm})$ are biosynthesized in the dark. Pr phytochromes can be phototransformed into biologically active, far-red light-absorbing (Pfr) phytochromes $(\lambda \max =680 \mathrm{~nm})$ upon exposure to red light. This photoactivation induces a highly regulated signaling network for photoresponses in plants, which may be one of most the important biochemical reactions on Earth, because we all depend on the light-harvesting machinery of plants. Seeking biotechnological applications for crop improvement, professor Kim's group used Arabidopsis to generate hyperactive phytochrome mutants, including 
hyperactive PhyA mutants, bathochromic phytochrome mutants, and constitutively active phytochrome mutants. The hyperactive PhyA mutants, lacking the phosphorylation sites involved in light-signal attenuation, elicited enhanced light responses and increased chlorophyll content. The Pr-absorption spectrum of the bathochromic phytochrome mutants was shifted to a longer wavelength than that of wild-type plants. Transgenic Arabidopsis plants bearing the bathochromic phytochrome exhibited shade-tolerant phenotypes and responses to far-red light that were approximately 100 -fold stronger than those of wild-type plants. The constitutively active phytochrome mutants showed constitutive photomorphogenic phenotypes in the dark, including the hook opening and shortened hypocotyls. The results demonstrated using these hyperactive phytochrome mutants show promise for engineering crops with enhanced agricultural performance in the future.

Professor Pil Joon Seo (Chonbuk National University, Korea) was the first speaker in the second session, giving a talk entitled "Peptide interference as a novel way of transcriptional control". Transcriptional regulation may be the most economical way to control gene expression. Transcription factors (TFs) are key components of transcriptional networks. TF activities are regulated at multiple steps, including transcription, posttranscriptional mRNA metabolism, posttranslational modification, and nucleocytoplasmic transport. Combinatorial TF dimerization plays a critical role in the diversification of the transcriptional specificities of TFs. A similar, but biochemically distinct, mechanism is competitive inhibition, whereby small proteins act as negative regulators by forming nonfunctional heterodimers with specific TFs. Small interfering peptides (siPEPs) possess a protein dimerization motif but lack the DNA-binding motif, forming nonfunctional heterodimers with specific TFs, resulting in transcriptional regulation. Extensive database searches for small proteins with a similar structural organization revealed that siPEP-mediated transcription control is a widespread regulatory mechanism in plants. Alternative splicing of a single TF gene produces multiple proteins with different structuraldomain compositions. Professor Seo explained that alternative splicing of some TF genes generates siPEPs that negatively regulate the target TFs via peptide interference, constituting self-regulatory circuits that act in plant development. Thus, siPEP is a newly emerging mechanism of transcriptional regulation with many potential biotechnological applications for crop improvement.

The last two speakers of the second session discussed brassinosteroids (BRs), a type of plant-steroid hormone. BRs play a broad range of essential roles in plant growth, development, stress adaptation, and defense. Professor Man-Ho Oh (Chungnam National University, Korea) presented a talk entitled "Functional importance of tyrosine phosphorylation in brassinosteroid signaling and defense mechanism". BR Insensitive 1 (BRI1) is a BR receptor. BRI1-associated kinase 1 (BAK1) is a co-receptor with BRI1. Both BRI1 and BAK1 are classified as Ser/Thr protein kinases. Professor Oh presented results on the dualspecificity of the cytoplasmic domains of BRI1 and BAK by autophosphorylation at the tyrosine residues. BAK1 is a leucine-rich repeat receptor-like kinase (LRR-kinase) that functions as a co-receptor with BRI1 and the flagellin receptor FLS2; it also functions as a negative regulator of programmed cell death. BAK1 is autophosphorylated at numerous serine/threonine sites; it transphosphorylates the associated receptor kinase and can also autophosphorylate at tyrosine residues. Professor Oh's group identified Tyr610 in the carboxy-terminal domain of BAK1 as a major autophosphorylation site. The expression of BAK1(Y610F)-Flag in transgenic plants lacking the endogenous $b a k l$ and its functional paralog $b k k l$ produced plants that were viable but extremely stunted and generally resembled BR signaling mutants. An acidic substitution for Tyr-610, to mimic phosphorylation, restored normal growth. Transcriptome analysis showed that many BRregulated genes were not properly expressed in the plants expressing BAK1(Y610)-Flag, suggesting that phosphorylation of Tyr-610 is essential for BR signaling but not for the suppression of cell death. In addition, the basal expression of many defense genes was dramatically reduced in the Y610F-directed mutants, implying that tyrosine phosphorylation may enhance the BAK1-mediated expression of many plant innate-immunity genes. The second part of Professor Oh's talk was on the glucosinolates (GSs). GSs are plant defense-related metabolites that generate bioactive compounds, isothiocyanates. Sitedirected mutation at Tyr-610 of BAK in Arabidopsis altered the methionine-derived aliphatic GS profiles and the expression of the GS hydrolysis-related genes epithiospecifier protein (ESP) and epithiospecifier modifier 1 (ESM1). Transgenic plants expressing BAK1(Y610F)-Flag displayed strongly diminished accumulation of 4-methylsulphinylbutyl GS and increased levels of 3-methylsulphinylpropyl GS. The changes in the aliphatic GSs of the transgenic plants resembled those of a methylthioalkylmalate synthase 1 ( $\mathrm{maml}$ ) knockout mutant. Expression of $E S P$ in BAK1(Y610F) plants was increased more than 50-fold, whereas that of ESM1 was decreased tenfold, suggesting that the outcome of the myrosinase-catalyzed GS hydrolysis was diverted towards the production of nitrils, which appear to be less toxic than isothiocyanates to herbivorous insects. Indeed, preference tests and larval feeding experiments with a herbivorous insect, Trichoplusia $n i$, showed higher feeding preference and larval weight gains among insects feeding on BAK1(Y610F) plants compared with those feeding on wild-type normal 
BR-signaling plants. Professor Oh's group is currently conducting experiments to identify the components interconnecting the function of site-specific Tyr phosphorylation in BAKl with those of the GS side-chain elongation enzyme NAM1 and ESP/ESM1 GS-hydrolysis factors.

The presentation of the last speaker, Professor Tae-Wuk Kim (Hanyang University, Korea), was entitled "Plant GSK3-mediated cell growth and development". Unlike the animal steroids, which function through nuclear receptors, BR binds to a receptor kinase (BRI1) at the cell surface to activate the BR-responsive TFs, BZR1 and BZR2. Although the complete BR signaling pathway (BR, BRI1, BSK1/CDG1, BSU1, BIN2, BZR1/BZR2) is established, the molecular connections between BR signaling and developmental outcomes are still largely unknown. Professor Kim presented results on stomatal development in association with BR signaling. Stomata are epidermal pores that control the acquisition of carbon dioxide for photosynthesis in plants. The development of stomatal cells is regulated by ERCTA family (ERf) receptor kinases and a mitogen activated protein kinase (MAPK) module. While genetic evidence suggests that the ERf and MAPK modules are in a common pathway, how upstream signaling regulates the MAPK module remains unclear. Professor Kim's experimental results show that BR regulates stomatal development through a branched pathway, whereby the GSK3-like kinase BIN2 regulates the MAP3K YDA. Genetic analyses indicate that the BR-mediated inhibition of stomatal development requires upstream BR-signaling components but is independent of the BR-transcription factor BZR1. Stomatal clustering caused by the disruption of ERf kinases is suppressed via the inhibition of BIN2, suggesting that BIN2 modulates the upstream signaling of MAPK. In vitro and in vivo assays show that BIN2 interacts with and phosphorylates YDA. Professor Kim's results reveal crosstalk between two major signaling pathways via GSK3-mediated regulation, providing a mechanism whereby BR coordinates plant development with elements of photosynthesis to promote optimal plant growth.

The author NSK is the editor-in-chief of Genes \& Genomics. 